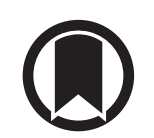

CrossMark

\title{
Post-approval upper airway stimulation predictors of treatment effectiveness in the ADHERE registry
}

\author{
Clemens Heiser ${ }^{1}$, Armin Steffen ${ }^{2}$, Maurits Boon ${ }^{3}$, Benedikt Hofauer ${ }^{1}$, \\ Karl Doghramji ${ }^{3}$, Joachim T. Maurer ${ }^{4}$, J. Ulrich Sommer ${ }^{4}$, Ryan Soose ${ }^{5}$, \\ Patrick J. Strollo Jr ${ }^{5}$, Richard Schwab ${ }^{6}$, Erica Thaler ${ }^{6}$, Kirk Withrow ${ }^{7}$, \\ Alan Kominsky ${ }^{8}$, Christopher Larsen ${ }^{9}$, Eric J. Kezirian ${ }^{10}$, Jennifer Hsia ${ }^{11}$, \\ Stanley Chia ${ }^{12}$, John Harwick ${ }^{13}$, Kingman Strohl ${ }^{14}$ and Reena Mehra ${ }^{8}$, on behalf \\ of the ADHERE registry investigators
}

Affiliations: ${ }^{1}$ Dept of Otorhinolaryngology, Head and Neck Surgery, Munich Technical University, Munich, Germany. ${ }^{2}$ Dept of Otorhinolaryngology, University of Lubeck, Lubeck, Germany. ${ }^{3}$ Dept of Otorhinolaryngology Head and Neck Surgery, Thomas Jefferson University, Philadelphia, PA, USA. ${ }^{4}$ Dept of Otorhinolaryngology, Head and Neck Surgery, HELIOS University Hospital Wuppertal, Wuppertal, Germany. ${ }^{5}$ Dept of Otorhinolaryngology, University of Pittsburgh, Pittsburgh, PA, USA. ${ }^{6}$ Dept of Sleep Medicine, University of Pennsylvania, Philadelphia, PA, USA. ${ }^{7}$ Dept of Otorhinolaryngology, University of Alabama, Birmingham, AL, USA. ${ }^{8}$ Dept of Otorhinolaryngology, Cleveland Clinic, Cleveland, OH, USA. ${ }^{9}$ Dept of Otorhinolaryngology - Head and Neck Surgery, Kansas University Medical Center, Kansas City, KS, USA. ${ }^{10}$ Dept of Otorhinolaryngology - Head and Neck Surgery, Keck School of Medicine of the University of Southern California, Los Angeles, CA, USA. ${ }^{11}$ Dept of Otorhinolaryngology - Head and Neck Surgery, University of Minnesota Fairview Hospital, Minneapolis, MN, USA. ${ }^{12}$ Dept of Otorhinolaryngology, MedStar Washington Hospital Center, Washington, DC, USA. ${ }^{13}$ Dept of Otorhinolaryngology - Head and Neck Surgery, University of Florida, Gainesville, FL, USA. ${ }^{14}$ Dept of Pulmonary and Critical Care Medicine and Sleep Medicine, University Hospital Cleveland Medical Center, Cleveland, OH, USA.

Correspondence: Clemens Heiser, Dept of Otorhinolaryngology, Head and Neck Surgery, Technische Universität München, Ismaninger Str. 22, 81675 Munich, Germany. E-mail: hnođheiser-online.com

@ERSpublications

In this largest international report to date, a substantial improvement in OSA severity in response to UAS is identified. Increasing age and reduced BMI are predictors of UAS. http://ow.ly/mtGa30mmTib

Cite this article as: Heiser C, Steffen A, Boon M, et al. Post-approval upper airway stimulation predictors of treatment effectiveness in the ADHERE registry. Eur Respir J 2019; 53: 1801405 [https://doi.org/10.1183/ 13993003.01405-2018].

ABSTRACT Upper airway stimulation (UAS) has been shown to reduce severity of obstructive sleep apnoea. The aim of this study was to identify predictors of UAS therapy response in an international multicentre registry.

Patients who underwent UAS implantation in the United States and Germany were enrolled in an observational registry. Data collected included patient characteristics, apnoea/hypopnoea index (AHI), Epworth sleepiness scale (ESS), objective adherence, adverse events and patient satisfaction measures. Post hoc univariate and multiple logistic regression were performed to evaluate factors associated with treatment success.

Between October 2016 and January 2018, 508 participants were enrolled from 14 centres. Median AHI was reduced from 34 to 7 events $\mathrm{h}^{-1}$, median ESS reduced from 12 to 7 from baseline to final visit at 12-month post-implant. In post hoc analyses, for each 1 -year increase in age, there was a $4 \%$ increase in odds of treatment success. For each 1-unit increase in body mass index (BMI), there was $9 \%$ reduced odds of treatment success. In the multivariable model, age persisted in serving as statistically significant predictor of treatment success.

In a large multicentre international registry, UAS is an effective treatment option with high patient satisfaction and low adverse events. Increasing age and reduced BMI are predictors of treatment response.

This article has supplementary material available from erj.ersjournals.com

Received: Aug 142018 | Accepted after revision: Oct 192018

The study was registered at www.clinicaltrials.gov as NCT02907398. Data not depicted in the manuscript are not made publicly available.

Copyright OERS 2019. This version is distributed under the terms of the Creative Commons Attribution NonCommercial Licence 4.0. 


\section{Introduction}

Obstructive sleep apnoea (OSA) is common disorder; one treatment target option is to activate the lingual muscles and open the upper airway [1]. A fall in genioglossal electromyography activity (innervated by the hypoglossal nerve (CN XII), the motor nerve to the tongue) can result in closure of a vulnerable region of the oropharynx, producing the obstructive apnoea, and activation reopens the airway $[2,3]$. Factors other than airway closure by muscle atonia will influence the frequency of respiratory events and severity of OSA; arousal threshold, loop gain and muscle responsiveness play an important role and could be untouched by hypoglossal nerve stimulation [4]. Repetitive periods of OSA produce nocturnal hypoxaemia and sleep fragmentation, and if left untreated, the disorder is associated with cognitive, behavioural and cardiovascular morbidities and an increase in all-cause mortality [5]. Current anatomical surgical procedures and mechanical treatments (continuous positive airway pressure (CPAP) therapy and oral appliances), although effective, are unsatisfactory for many patients, or are accompanied by poor adherence, leaving a significant number of untreated patients with moderate-to-severe disease [6].

Upper airway stimulation (UAS; Inspire Medical Systems, Maple Grove, MN, USA) is a system of unilateral hypoglossal nerve stimulation, consisting of an implantable pulse generator, stimulation lead placed on the hypoglossal nerve and respiratory sensing electrode. The European (2009) and United States Food and Drug Administration (FDA) (2014) approvals were based upon demonstration of effectiveness and safety for selected patients with moderate-to-severe OSA who failed or were intolerant to positive airway pressure therapy [7, 8]. Therapy directions from phase II studies and the phase III STAR (Stimulation Therapy for Apnea Reduction) trial indicate better success for those with an apnoea/ hypopnoea index (AHI) of $15-65$ events $\cdot \mathrm{h}^{-1}$, a body mass index (BMI) generally $<35 \mathrm{~kg} \cdot \mathrm{m}^{-2}$ and an "appropriate" anatomy [9, 10]. Anatomical evaluation was based largely on the absence of complete concentric collapse at the level of the velopharynx during drug-induced sleep endoscopy (DISE) examination. Surgical training is needed for optimal electrode placement on the distal and medial branches of CN XII. Following the placement, a period of optimisation of home therapy with the stimulator, including follow-up verification of its effectiveness is accomplished $[11,12]$.

However, controlled protocols such as the STAR trial may not translate well into clinical practice. Deployment beyond the trial centres opens the opportunity for the examination of variations in patient referral, financial resources, safety and adherence. In addition, variations in implantation, management by different specialists and differences in insurance reimbursement may influence outcomes. The ADHERE (Adherence and Outcome of Upper Airway Stimulation for OSA International Registry) registry was designed to monitor this transition from trial to clinical practice. The ADHERE platform was created to collect demographic, surgical outcome, complications, quality of life and patient-reported outcomes undergoing treatment with UAS in the United States and Europe. ADHERE is a cumulative cohort, designed to follow the progress of implementation across many centres and across time in the provision of this technology. The primary purpose of this report is to characterise the predictors of responsiveness and adherence of therapy use in this post-approval cohort. In addition, we describe safety and effectiveness in the largest cohort to undergo UAS available to date.

\section{Methods}

\section{Study design}

The registry was approved by ethics committees or institutional review boards of every implant centre. The study was registered with www.clinicaltrials.gov (NCT02907398). All procedures followed were in accordance with the ethical standards of the responsible committee on human experimentation (institutional and national) and with the Helsinki declaration of 1964 and later revision. Informed consent was signed by every patient. Ongoing patients, who received a UAS implant in all participating implant centres in the USA and Germany and who agreed to participate were included in this multicentre, prospective and retrospective observational registry. The aim was not to change the clinical routine of the patient's treatment. The registry is non-interventional, and no study-specific procedures were added. Adult patients with a moderate to severe degree of OSA (AHI $15-65$ events. $\mathrm{h}^{-1}$ ) and an intolerance to CPAP, who underwent an implantation of a UAS system (Inspire II and IV) were included. CPAP intolerance was defined by clinical criteria, namely if the patient was unable or unwilling to use CPAP. In the United States and Europe, CPAP intolerance is defined as 1 ) inability to use CPAP ( $>5$ nights per week of usage, usage defined as $>4 \mathrm{~h}$ of use per night) or 2) unwilling to use CPAP (for example, a patient returns the CPAP system after attempting to use it or has claustrophobia on repeated use). As already described in previous studies, favourable anatomical criteria were also considered in the decision to implant [9].

\section{Procedures and sleep recordings}

The UAS system consists of a cuff electrode to stimulate the medial branches of the hypoglossal nerve, which activates the protrusor tongue muscles to open the upper airway; a pressure sensor, which is responsible for 
detecting the breathing cycles, with its lead is placed within the fourth or fifth intercostal space; and an implantable pulse generator inserted into a subcutaneous pocket $\sim 4 \mathrm{~cm}$ below the clavicle [11]. The details of the surgery are described in prior publications [11-13]. The system and implant are intended to stimulate upper airway opening by hypoglossal nerve fibres, ideally during inspiration, in order to protect the airway from obstruction during expiration.

The system is activated with standard settings programmed $\sim 1$ month after the surgical procedure, after an interim surgical follow-up. During the next 4 weeks or so, patients are asked to gradually increase the stimulation amplitude. This period allows participants to experience therapy and personally optimise comfort and subjective effectiveness according to preset limits. Subsequent to this adjustment period, symptoms and comfort with UAS are assessed. In many centres, patients had an in-lab polysomnographic (PSG) titration study 2-6 months after implant to optimise therapy. In some centres, for insurance purposes, portable studies were undertaken to confirm settings or determine the need for further adjustments. Following the therapeutic titration, patients were seen in the office for follow-up, and symptoms, adverse events and stimulation thresholds were assessed, along with the device settings. The post-titration visit, in many cases, was the first office visit after system set-up and titration. The final ADHERE visit was to occur $\sim 12$ months post-implantation.

Information that was collected included the baseline AHI prior to UAS implantation, the treatment AHI post-titration and the AHI at final visit. The treatment AHI, or AHI measured under the therapeutic setting, was assessed during a home sleep apnoea test (HST), an in-lab PSG, during the initial titration PSG or during an additional titration PSG. Respiratory event index for HST and AHI for PSG were summarised under the term "AHI". When the AHI was determined during a PSG, the AHI ("treatment AHI”) was collected, which was under therapeutic settings for this time period during the night. The objective outcomes of AHI and oxygen desaturation index were scored using standard 2007 scoring criteria in most centres, with hypopnoea scored based upon $30 \%$ airflow reduction and a $4 \%$ oxygen desaturation [14]. The registry did not specify AHI scoring criteria to follow, because the aim of the study is to assess how therapy effectiveness is being assessed in clinical practice. As in other studies examining surgical success of OSA treatment, we defined effective OSA treatment as a reduction of AHI by $\geqslant 50 \%$ to $\leqslant 20$ events $\cdot h^{-1}$ [15]. Additionally, treatment duration was collected in hours of use per night, provided by interrogation of the implantable pulse generator (IPG) with a programmer at the post-titration visit.

The Epworth sleepiness scale (ESS) was administered at baseline, post-titration and at the final visit. A custom-designed survey for the patient's experience with therapy was provided. At every visit, the Clinical Global Impression - Improvement (CGI-I) was completed by the physician to provide an estimate of how much the patient's disease had improved or worsened compared to baseline before initiating treatment.

Adverse events were monitored from implant until the last follow-up visit. This was done to collect data relevant to procedure and device safety. A reportable adverse event included any event related or possibly related to the implant procedure or stimulation therapy that occurs at a level, intensity or time frame greater than expected. Adverse event severity was classified as mild (aware of event, but easily tolerated), moderate (discomfort enough to cause interference with usual activity) or severe (inability to carry out usual activity).

\section{Statistical analysis}

Outcome measures of AHI and ESS from the follow-up visit were compared to the baseline measurement. A paired t-test was used to evaluate the difference between baseline and follow-up visit with a type I error rate of 0.05 . Results are presented as median and mean \pm SD. Post hoc logistic regression analyses include a univariate model of all potential predictors. An additional multivariate model with stepwise selection was used to retain only significant parameters for assessing for predictors of the therapy. In addition, adherence was performed with data from the final visit. Odds ratios were calculated for the different parameters with a p-value $<0.05$ considered as statistically significant.

\section{Results}

Between October 2016 and January 2018, 508 participants were enrolled from 14 centres. Three centres had previous experience with UAS in the initial STAR trial; the remaining 11 were new to implementing UAS. The study cohort consisted of a middle-aged, primarily male (79\%), Caucasian (97\%) and overweight population. Overall, there was no systolic hypertension in this group. However, diastolic hypertension was present with a mean diastolic blood pressure of $78 \pm 9 \mathrm{mmHg}$ (table 1).

The average surgical time, available from 429 reported implant procedures, was $142 \pm 45 \mathrm{~min}$. The most common tongue motion accomplished during intra-operative testing was bilateral protrusion (70\%), right protrusion $(21 \%)$ and others $(9 \% ; 38$ cases of other tongue motion including left-protrusion or retraction). 


\section{TABLE 1 Baseline characteristics of the registry participants}

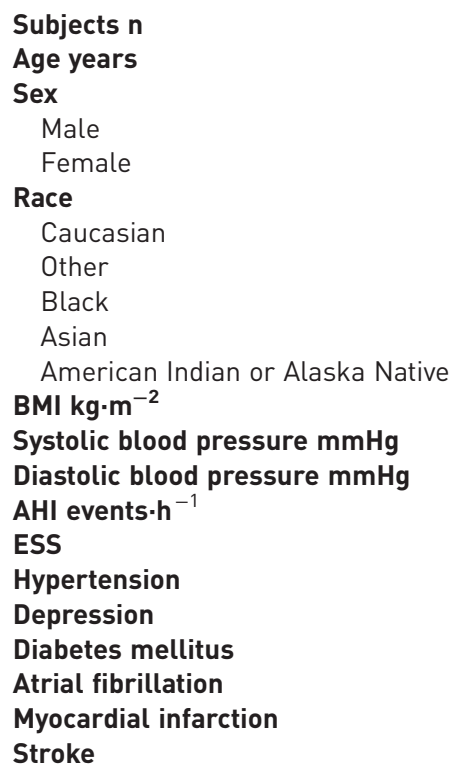

Data are presented as mean \pm SD, $\mathrm{n}(\%)$ or \%, unless otherwise stated. BMI: body mass index; AHI: apnoea/ hypopnoea index; ESS: Epworth sleepiness scale.

The majority $(88 \%)$ of United States patients were discharged on the same day, while all patients stayed overnight in the German centres.

\section{Post-titration outcomes}

Post-titration patient outcome was assessed $137 \pm 77$ days after UAS implant. The mean BMI did not change from baseline to post-titration follow-up $\left(29.3 \pm 3.9 \mathrm{~kg} \cdot \mathrm{m}^{-2}\right.$ to $\left.29.2 \pm 4.1 \mathrm{~kg} \cdot \mathrm{m}^{-2}, \mathrm{p}>0.05\right)$. The mean AHI decreased significantly from baseline of $36.3 \pm 15.7$ events $\cdot \mathrm{h}^{-1}$ to $10.2 \pm 13.3$ events $\cdot \mathrm{h}^{-1}$ at post-titration $\left(\mathrm{p}<0.0001\right.$; figure 1) and median AHI decreased from 34.0 events $\cdot \mathrm{h}^{-1}$ to 5.7 events $\cdot \mathrm{h}^{-1}$. The absolute AHI reduction from baseline was $-25.7 \pm 16.5$ events $\cdot h^{-1}$ and a relative reduction of $72 \pm 32 \%$ was noted. AHI decreased by $\geqslant 50 \%$ to $\leqslant 20$ events $\mathrm{h}^{-1}$ in $92 \%$ of patients. At post-titration, AHI $\leqslant 5 \mathrm{events} \cdot \mathrm{h}^{-1}$, $\leqslant 10$ events $\mathrm{h}^{-1}$ or $\leqslant 15$ events. $\mathrm{h}^{-1}$ was achieved in $53 \%, 79 \%$ and $94 \%$ of patients, respectively. ESS decreased significantly from baseline of $11.8 \pm 5.5$ to $7.7 \pm 4.9$ at post-titration $(p<0.0001)$ with median ESS

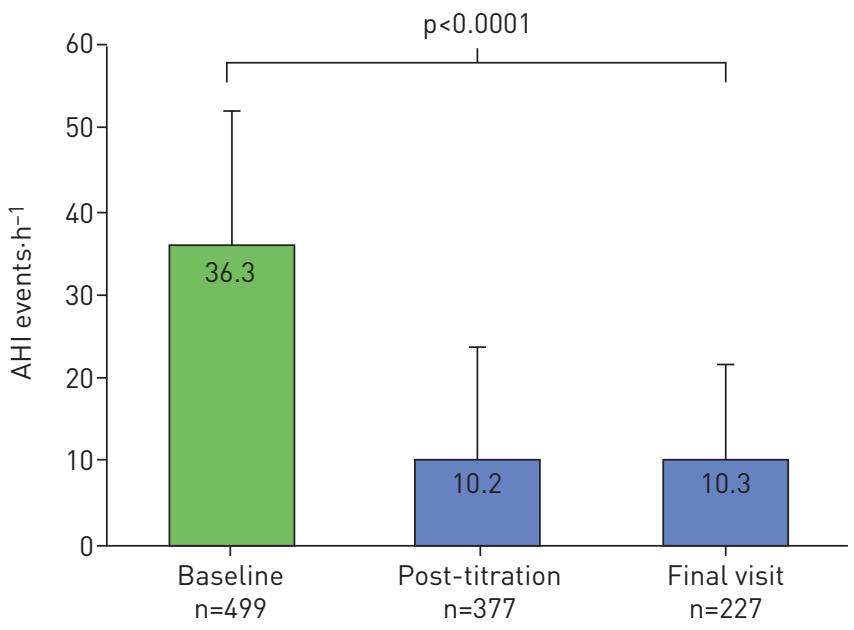

FIGURE 1 Changes in apnoea/hypopnoea index (AHI) from baseline to post-titration and final visit results. Data are presented as mean+sD. 


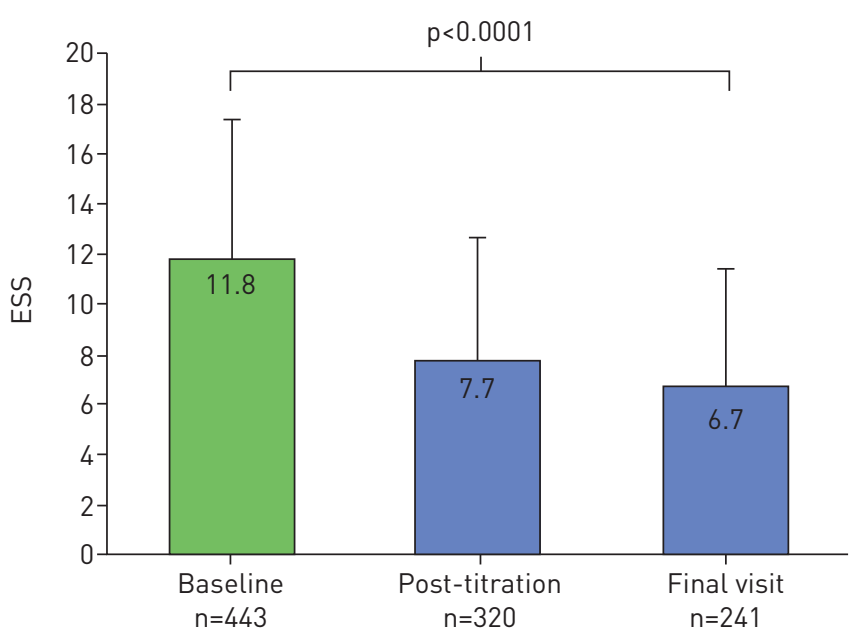

FIGURE 2 Changes in Epworth sleepiness scale (ESS) from baseline to post-titration and final visit results. Data are presented as mean+sD

decreasing from 12 to 7 (figure 2). The proportion of patients with ESS $<10$ increased from $37 \%$ to $65 \%$ from baseline to post-titration.

\section{Final visit outcomes}

The final visit adherence outcome was assessed 386 \pm 136 days after UAS implant. The mean BMI did not significantly change from baseline to the final visit $\left(29.3 \pm 3.9 \mathrm{~kg} \cdot \mathrm{m}^{-2}\right.$ to $\left.28.9 \pm 4.2 \mathrm{~kg} \cdot \mathrm{m}^{-2}, \mathrm{p}>0.05\right)$. The mean AHI decreased significantly from baseline of $36.3 \pm 15.7$ events $\cdot h^{-1}$ to $10.3 \pm 11.5$ events $\cdot h^{-1}$ at final visit $(\mathrm{p}<0.0001)$ with the median AHI reduced from 34.0 events $\cdot \mathrm{h}^{-1}$ to 7.0 events $\cdot \mathrm{h}^{-1}$ (figure 1 ). The absolute AHI reduction from baseline was $-24.3 \pm 17.3$ events $\cdot \mathrm{h}^{-1}$ and a relative reduction of $68 \pm 34 \%$. AHI reduced by $\geqslant 50 \%$ to $\leqslant 20$ events $\cdot \mathrm{h}^{-1}$ in $81 \%$ of patients. At post-titration, AHI $\leqslant 5 \mathrm{events} \cdot \mathrm{h}^{-1}$, $\leqslant 10$ events. $\mathrm{h}^{-1}$ or $\leqslant 15$ events. $\mathrm{h}^{-1}$ was achieved in $41 \%, 65 \%$ and $78 \%$ of patients, respectively. ESS decreased significantly from a baseline of $11.8 \pm 5.5$ events $\cdot h^{-1}$ to $6.7 \pm 4.7$ events $\cdot h^{-1}$ at final visit $(\mathrm{p}<0.0001)$ with median ESS reduced from 12 to 6 . Patients with ESS $<10$ increased from $37 \%$ to $76 \%$ from baseline to final visit (figure 2).

$94 \%$ of physicians rated syndromic improvement on the CGI-I relative to baseline prior to UAS implant at post-titration, which persisted at $93 \%$ at final visit. Of note, $48 \%$ of physician noted a high degree of improvement post-titration that increased at the final visit to $57 \%$ (figure 3). Regarding patient-reported responses to therapy experience, $96 \%$ of patients reported that UAS was better than as remembered CPAP therapy at post-titration and at the final visit, with $95 \%$ stating that they would undergo UAS again at the post-titration visit, and $94 \%$ at the final visit. At the post-titration visit, $93 \%$ of patients reported that they would recommend UAS to family and friends, which increased to $96 \%$ at the final visit. At the post-titration visit, $91 \%$ of patients reported that overall they were satisfied with UAS therapy; at the final visit it was $94 \%$ (figure 4 ).

\section{Overview of safety outcomes}

The majority (98\%) of the 508 implanting procedures were completed without a report of an adverse event. There were two cases of intra-operative bleeding during the tunnelling of the stimulation lead; both stopped upon application of pressure. Two cases of seroma both resolved without sequelae. One patient was found with submandibular swelling, two patients with transient tongue weakness and three patients with transient dysarthria. One patient was found with a dislodged stimulation cuff at the activation visit 1 month post-implant, which was repositioned without complications. At the post-titration visit, a total of 87 adverse events were reported in $23 \%$ of patients. At the final visit, a total of 61 adverse events were reported in $23 \%$ of patients (table 2). One patient underwent a revision procedure to reposition the cuff electrode due to inadequate therapy response. The revision procedure was completed without complications. The AHI of the patients with a revision was 28.3 events $\cdot \mathrm{h}^{-1}, 24$ events $\cdot \mathrm{h}^{-1}$ and 14 events $\cdot \mathrm{h}^{-1}$ at the baseline, post-titration and final visit, respectively. The definitions of ratings of mild, moderate and severe adverse events varied across sites. The details of events across categories and those categorised as severe are provided in online supplementary tables S2 and S3, respectively. 


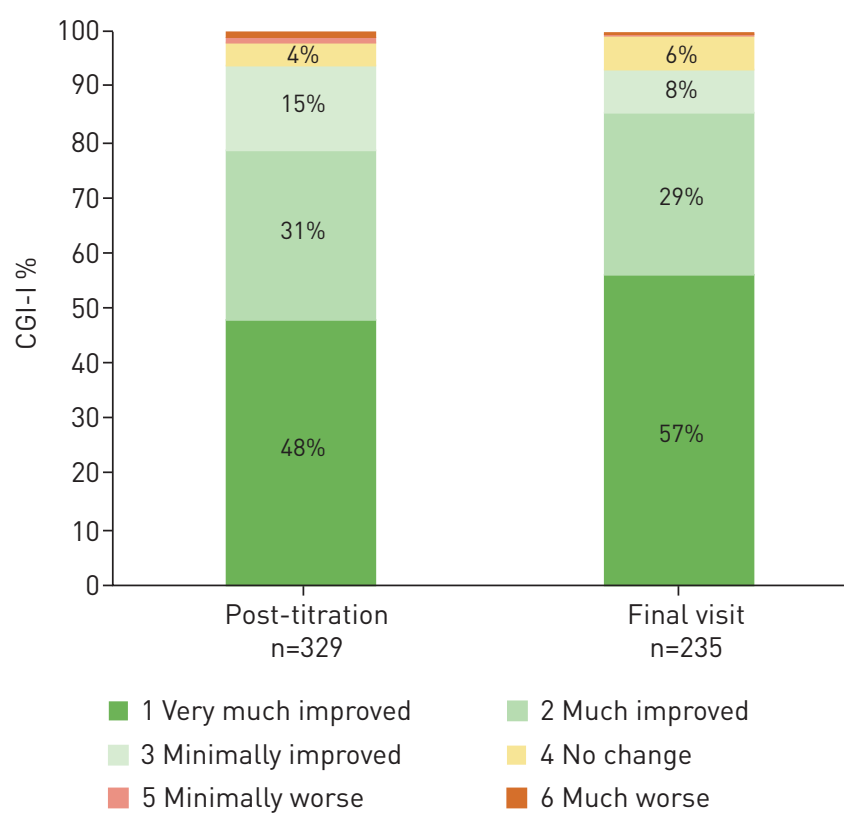

FIGURE 3 Clinical Global Impression of the Intervention - Improvement (CGI-I) rated by the physician at post-titration and final visit.

Primary results: predictors of upper airway stimulation effective treatment and adherence In the univariate logistic regression analysis, age and body mass index were significantly associated with OSA treatment success. Specifically, for each 1-year increase in age, there was a $4 \%$ increase in odds of OSA treatment success. Alternatively, for each 1-unit increase in BMI, there was $9 \%$ reduced odds of OSA

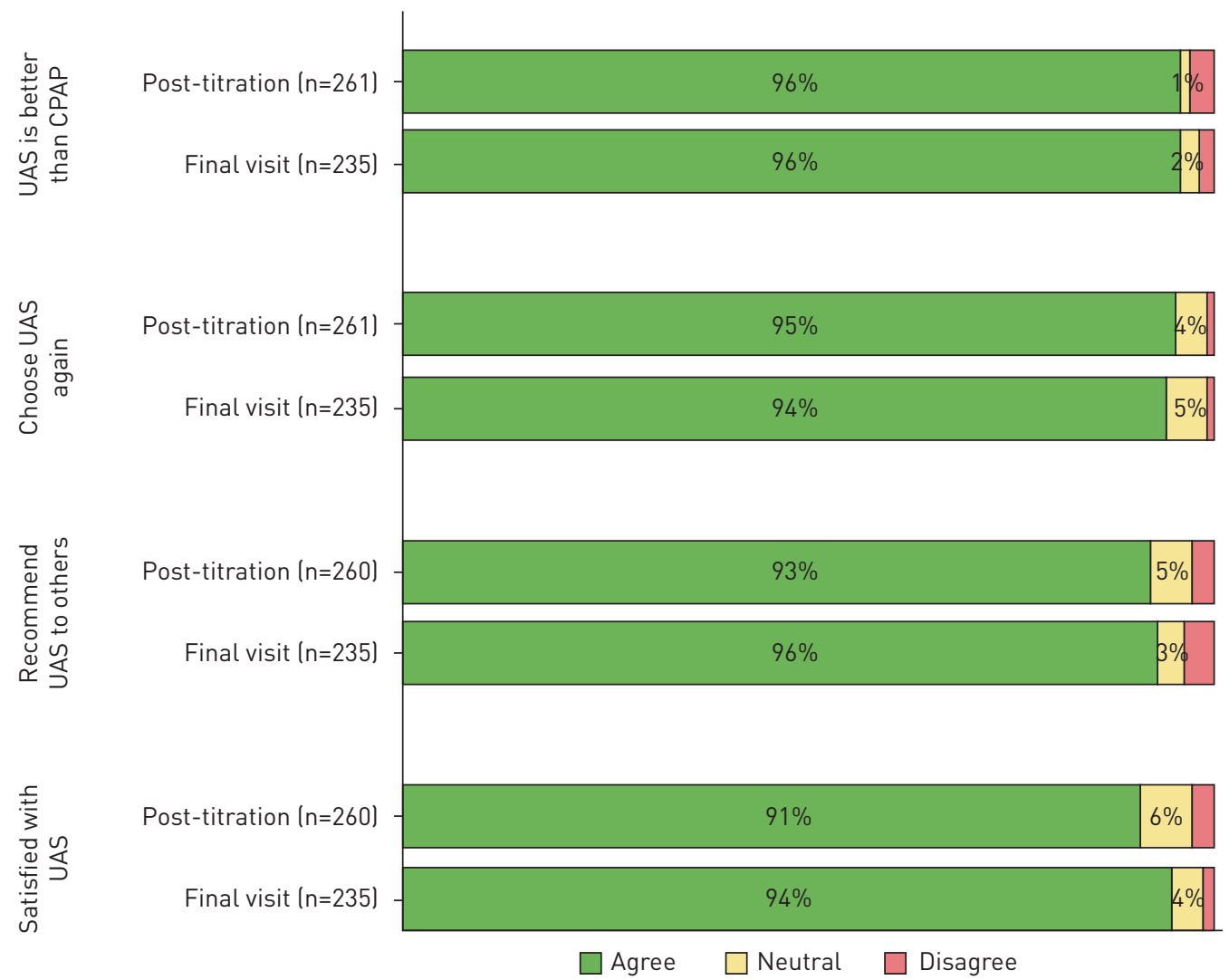

FIGURE 4 Patient experience with upper airway stimulation (UAS) at the post-titration and final visits. CPAP: continuous positive airway pressure. 
TABLE 2 Summary of post-operative adverse events

\begin{tabular}{|c|c|c|c|c|}
\hline & \multicolumn{2}{|c|}{ Post-titration } & \multicolumn{2}{|c|}{ Final visit } \\
\hline & $\begin{array}{c}\text { Events } \\
n\end{array}$ & $\begin{array}{c}\text { Patients } \\
\%\end{array}$ & $\begin{array}{c}\text { Events } \\
n\end{array}$ & $\begin{array}{c}\text { Patients } \\
\%\end{array}$ \\
\hline Tongue weakness & 2 & $<1$ & 0 & \\
\hline Swallowing or speech related & 3 & $<1$ & 0 & \\
\hline Discomfort (incision/scar) & 5 & 1 & 5 & 2 \\
\hline Discomfort (device) & 7 & 2 & 3 & 1 \\
\hline Infection & 0 & & 0 & \\
\hline Post-operative - other ${ }^{\#}$ & 8 & 2 & 3 & 1 \\
\hline Stimulation-related discomfort & 26 & 8 & 15 & 6 \\
\hline Tongue abrasion & 7 & 2 & 7 & 3 \\
\hline Insomnia/arousal & 6 & 2 & 9 & 3 \\
\hline Revision interventions (including explant) & 0 & & 1 & $<1$ \\
\hline Other discomfort & 5 & 1 & 2 & $<1$ \\
\hline Activation - other & 18 & 5 & 16 & 6 \\
\hline Total & 87 & 23 & 61 & 23 \\
\hline
\end{tabular}

72 patients reported adverse events at the post-titration visit and 50 at the final visit (not mutually exclusive). Some patients reported multiple adverse events. Percentage of patients was calculated based on the number of patients at each visit who completed the visit form, which contains adverse event information. \#: includes shortness of breath, seroma, numbness of the throat and hoarseness during the day and a mild tongue-base and epiglottic obstruction.

treatment success (table 3). In the multivariable model, only age persisted in serving as a statistically significant predictor of UAS OSA treatment success. The degree of OSA at baseline and hours of usage were not significant predictors of success (table 3 ). That means that patients who used their device more than other patients did not show a better chance of a successful treatment. Interestingly, although not statistically significant, females had a point estimate reflecting a nearly three-fold higher odds of OSA treatment success with UAS compared to males.

The objective adherence monitoring was interrogated from the IPG and showed an average home device use of $6.4 \pm 2.0 \mathrm{~h}$ per night at post-titration $(n=344)$ and $5.7 \pm 2.2 \mathrm{~h}$ per night at final visit $(n=229)$. In terms of examination of predictors of UAS adherence, this was defined as usage for $>4 \mathrm{~h}$ per sleep period and alternatively defined as hourly average usage (table 4). Age was associated with increased adherence, such that for each year increase, there was a $9 \%$ increased odds ratio of UAS usage. Similar to the UAS success findings, BMI was a predictor of lower UAS adherence, such that for each unit increase in BMI, the odds of UAS adherence was $10 \%$ lower. Similarly, for each unit increase in baseline AHI, there was a $2 \%$ lower odds of UAS adherence. None of the parameters at the post-titration visit (such as tongue protrusion parameters) other than therapy use was associated with adherence at the final visit. A multivariable model using stepwise selection was fit with all parameters with univariable $p<0.20$ retaining only those with $p<0.05$. The final model included baseline AHI $(p=0.03)$ and therapy hours at final visit $(p<0.0001)$. With therapy hours, higher therapy hours at post-titration are associated with adherence at the final visit, while

TABLE 3 Logistic regression univariate analysis of predictors of obstructive sleep apnoea treatment success at the final visit (median time 373 days)

\begin{tabular}{llc} 
& OR $(95 \% \mathrm{Cl})$ & p-value \\
\hline Age (baseline) years & $1.04(1.01-1.08)$ & 0.01 \\
Sex (female versus male) & $2.62(0.88-7.78)$ & 0.08 \\
BMI (baseline) $\mathbf{k g} \cdot \mathbf{m}^{-2}$ & $0.91(0.83-0.99)$ & 0.03 \\
AHI (baseline) events.h & -1 & 0.88 \\
Therapy hours per week at post-titration visit & $1.00(0.98-1.03)$ & 0.25 \\
Therapy hours per week at final visit & $1.04(0.99-1.67)$ & 0.22 \\
Bilateral protrusion versus right protrusion & $1.02(0.99-1.04)$ & 0.37 \\
Other versus right protrusion & $2.08(0.96-4.51)$ & 0.61
\end{tabular}

A multivariable model was fit with stepwise selection used to reduce the model to retain only significant parameters (age and body mass index (BMI)) and only age was retained. AHI: apnoea/hypopnoea index. 
TABLE 4 Logistic regression of univariate analysis to assess for predictors of upper airway stimulation adherence ( $\geqslant 28 \mathrm{~h}$ per week) at the final visit

\begin{tabular}{lcc} 
& \multicolumn{1}{c}{ Univariable results } \\
\cline { 2 - 3 } & OR (95\% CI) & p-value \\
\hline Age at consent & $1.04(1.01-1.07)$ & 0.01 \\
Sex (female versus male) & $2.14(0.79-5.77)$ & 0.14 \\
BMI at baseline & $0.90(0.83-0.97)$ & 0.009 \\
Baseline AHI & $0.98(0.96-0.999)$ & 0.04 \\
Bilateral protrusion versus right protrusion & $0.92(0.41-2.11)$ & 0.59 \\
Other versus right protrusion & $1.33(0.32-5.59)$ & 0.62 \\
Change in AHI at post-titration visit & $1.01(0.99-1.04)$ & 0.19 \\
Change in ESS at post-titration visit & $0.98(0.91-1.05)$ & 0.57 \\
AHI success at post-titration visit & $0.99(0.42-2.34)$ & 0.98 \\
Improvement by CGI at post-titration visit & $0.67(0.30-1.51)$ & 0.33 \\
Patient satisfied or strongly satisfied at post-titration & $0.60(0.15-2.47)$ & 0.48 \\
Therapy use at post-titration & $1.08(1.05-1.11)$ & $<0.0001$ \\
Therapy use $\geqslant 28$ h per week at post-titration & $0.19(0.07-0.48)$ & 0.0004
\end{tabular}

A multivariable model was fit with stepwise selection used to reduce the model to retain only significant parameters (age, body mass index (BMI), apnoea/hypopnoea index (AHI) and therapy at post-titration) and only BMI and therapy use at post-titration visit was retained. ESS: Epworth sleepiness scale; CGI: Clinical Global Impressions scale.

higher baseline AHI is associated with less adherence at the final visit. Furthermore, there was no statistically significant association between sex of participants and treatment adherence.

\section{Discussion}

In this largest assembled cohort to date of patients undergoing UAS therapy, we identified a positive association for age, and an inverse association for BMI as predictors of UAS therapy effectiveness, as well as a trend towards females having increased odds of UAS therapy effectiveness. Objective adherence was high with an average usage of $5.7 \pm 2.2 \mathrm{~h}$ per day at the final visit. Age and BMI were positive and negative predictors, respectively, of final visit adherence [16]. These findings were no longer statistically significant in the multivariable model for BMI. The baseline AHI demonstrated a somewhat unanticipated inverse association with final visit adherence. The post-titration adherence (a marker of early adherence) was predictive of final visit adherence. These findings persisted in the multivariable model. Overall, these findings are of clinical significance to inform risk stratification and adherence optimisation strategies in those being considered for UAS.

UAS therapy resulted in an improvement in OSA severity (AHI $36.3 \pm 15.7$ events.h ${ }^{-1}$ to 10.3 \pm 11.5 events $\cdot h^{-1}$ ), comparable to STAR trial AHI outcomes. This was paralleled by improvement in daytime sleepiness. Physician clinical global impression scores were very high $(>90 \%)$ and patients rated UAS therapy higher than CPAP (i.e. would recommend to family and friends and were overall satisfied with therapy) with many of these measures increasing from post-titration to the final visit. The percentage of adverse events were low (2\%) and although some adverse events were rated as severe $(\mathrm{n}=9,6 \%$ of adverse events) by the individual sites, these did not meet criteria of serious adverse events as defined by the overall study.

Based upon findings from this post-approval registry, age and BMI were positive and negative predictors, respectively, of UAS effectiveness in OSA defined as a reduction of the AHI by $\geqslant 50 \%$ and $\mathrm{AHI}$ $<20$ events. $h^{-1}$ [15]. Upper airway surgical success predictors have been limited to pharyngeal classification, e.g. different rates of uvulopalatopharyngoplasty success, i.e. 52\% for type 1 (retropalatal obstruction alone) versus 5\% for types II (both retropalatal and hypopharyngeal obstruction) and III (hypopharyngeal obstruction alone) with few data on predictiveness of subject characteristics on success, mainly due to limited sample size [15]. In regard to age effect, the rationale behind the novel finding of enhanced UAS effectiveness in those who are older is not readily apparent. There may be ascertainment and referral bias, but it is interesting to speculate about mechanisms if one can assume that these factors are similar in older and younger patients who meet inclusion criteria.

There are ageing-related decreased collagen and elastin composition in the hyoepiglottic ligament [17, 18], so that nerve-muscular activation could have an enhanced effect. These ageing-related changes may enhance laxity of the ligament, thereby predisposing to posterior displacement, i.e. anteroposterior epiglottis-related obstruction [18]. Ageing can influence upper airway tissue structural integrity, conferring 
an increased risk of anteroposterior airway collapse. If tongue strength of older people is decreased, due to the fact that ageing leads to neuromuscular alterations of the tongue [19], one can envision that hypoglossal stimulation might be useful in CPAP-intolerant patients. Other possibilities include ageing-related behavioural factors, for instance seeking a technological innovation. The finding of increasing BMI associated with decrements in UAS effectiveness in OSA is consistent with initial feasibility studies $[20,21]$. DISE plays a crucial part in the inclusion criteria of UAS. It is known that with a higher BMI a higher probability of complete concentric palatal collapse occurs [22]. There is a grey zone, where the evaluating physician needs to decide if the collapse patterns during DISE are completely concentric or not. In advanced specialised centres, carefully selected patients even with a BMI $>35 \mathrm{~kg} \cdot \mathrm{m}^{-2}$ can still respond to therapy with good clinical outcomes [23].

The adherence in UAS at the final visit is highly favourable relative to adherence rates for CPAP. CPAP non-adherence estimates range between $29 \%$ and $83 \%$, mainly from clinic-based studies [24, 25]. The most consistent predictors of positive airway pressure (PAP) adherence include increasing age [24], early PAP adherence [16], self-reported daytime sleepiness [26] and certain psychological factors [27]. Although the prevalence of OSA is two- to four-fold higher in males, data focused upon the influence of sex on PAP adherence in OSA have been somewhat inconsistent $[28,29]$. Consistent with existing data on CPAP adherence data, increasing age was associated with increased odds of UAS adherence, suggesting factors inherent to the ageing process which transcend OSA therapy type contribute to enhanced usage. These findings demonstrate anticipated parallels of UAS success and adherence relative to increasing age. Conversely, increasing BMI was associated with reduction in UAS adherence, again consistent with UAS effectiveness findings. It is possible that the more limited impact on OSA treatment control in those who are more obese may result in a negative impact on adherence. Given the Caucasian predominance, we are limited in examination of race-specific differences of adherence. Although there was suggestion that UAS adherence may be greater in females than males, the smaller number of females in the registry may have precluded sufficient power to detect statistical significance. Given the borderline significant (albeit high magnitude of point estimate) findings showing increasing UAS responsiveness of females versus males, we cannot exclude the possibility of post-menopausal status with increased age as a potential explanatory factor. However, the $21 \%$ representation of females in this cohort is higher in the US FDA studies and is an area to examine more carefully as this registry increases in numbers and granularity of outcomes. Finally, similar to CPAP adherence, post-titration UAS adherence predicted final visit adherence, suggesting that early adopters are more likely to have durability of adherence.

The major strength of this study is the large sample size of individuals undergoing UAS therapy $(\mathrm{n}=508$ compared to the STAR trial [7] with $\mathrm{n}=126$ ) to date, which enables more effective examination of predictors of treatment success and adherence. Other strengths include the multicentre and multinational (United States and Europe) representation of patients included in the registry, and its clinic-based nature, which enables the examination of real-world effectiveness of UAS therapy implementation. Limitations include the use of post hoc analysis to identify predictors of treatment success and adherence, which need validation in prospective studies. Another limitation is that therapeutic AHI was gathered by different methods for the post-titration outcome visit, including PSG and HST and titration studies, where AHI may not represent the entire night value. In addition, the final visit adherence data were limited to a subsample given the ongoing nature of registry data collection. Furthermore, there are constraints in terms of limited sex and race diversity and distribution which precludes effective examination of these specific attributes in terms of UAS effectiveness and adherence. The lack of standardisation of scoring of the hypopnoea events across patients represents another limitation, although observed findings are likely to be similar, even with uniformity in hypopnoea scoring, because the same criteria were used at each centre for scoring the studies before and after UAS implantation.

Future investigations should be focused on elucidation of the mechanistic underpinnings underlying the age-related tissue and physiological upper airway alterations predisposing to enhanced amenability of UAS therapeutic benefit. A better understanding of how adiposity influences upper airway function and responsiveness to neurostimulation might lead to a reappraisal of BMI inclusion criteria to one more based on anatomy. Finally, existing knowledge gaps of sex- and race-specific differences in UAS treatment responsiveness and adherence should be better characterised by intentional augmentation of the registry with a more diverse sample of participants.

\section{Conclusion}

The international ADHERE registry reveals the effectiveness of UAS in a clinical setting in patients with OSA, who are noncompliant to CPAP. Furthermore, we identified a positive association for age, and an inverse association for BMI, as predictors of UAS therapy effectiveness and a trend towards females having an increased odd of UAS therapy effectiveness. 
Acknowledgements: We are in debt to all participants in the ADHERE registry for supporting data collection in all study visits.

Author contributions: C. Heiser, A. Steffen, M. Boon, B. Hofauer, K. Doghramji, J.T. Maurer, J.U. Sommer, R. Soose, P.J. Strollo Jr, R. Schwab, E. Thaler, K. Withrow, A. Kominsky, C. Larsen, E.J. Kezirian, J. Hsia, S. Chia, J. Harwick, K. Strohl and R. Mehra were involved in acquisition, analysis, interpretation of the data, and drafting and approval of the final manuscript.

Conflict of interest: C. Heiser reports grants and personal fees from Inspire Medical Systems (Maple Grove, MN, USA), during the conduct of the study; and grants and personal fees for consultancy from Inspire Medical Systems, outside the submitted work. A. Steffen has received reimbursement of conference fees and travel and accommodation expenses, fees for preparing scientific continuing professional development or other events, funding for research he himself initiated, and fees for conducting clinical studies on related subjects from Inspire Medical Systems. M. Boon reports grants from Inspire Medical Systems, during the conduct of the study; and grants and personal fees for consultancy from Inspire Medical Systems, outside the submitted work. B. Hofauer reports personal fees from Inspire Medical Systems, during the conduct of the study. K. Doghramji reports grants from Inspire Medical Systems, during the conduct of the study; and grants and personal fees from Inspire Medical Systems, outside the submitted work. J.T. Maurer reports grants and personal fees for lectures, surgical training and consulting from Inspire Medical Systems, during the conduct of the study; and grants and personal fees for surgical training from Imthera and Revent, grants and personal fees for consultancy from Philipps and Nyxoah, and personal fees for lecturing from GlaxoSmithKline, Heinen und Löwenstein, Weinmann, Sissel Novacare, ResMed, Olympus, Neuwirth Medical and Medtronic, outside the submitted work. J.U. Sommer reports grants, personal fees and non-financial support from Inspire Medical Systems, ImThera, Regis Medical, Nyxoah, Phillips, MedEl, and personal fees from Neucomed, during the conduct of the study. R. Soose was a consultant and study investigator for Inspire Medical Systems, during the conduct of the study; and carried out advisory board and consultancy work for Galvani Bioelectronics, and was a consultant for Invicta Medical, outside the submitted work. P.J. Strollo Jr reports grants and personal fees for consultancy from Inspire Medical Systems and Jazz Pharmaceuticals, and personal fees for consultancy from Philips-Respironics and Itamar Medical, outside the submitted work. R. Schwab reports grants from Inspire Medical Systems, during the conduct of the study. E. Thaler is a consultant for Inspire Medical Systems, outside the submitted work. K. Withrow has nothing to disclose. A. Kominsky has nothing to disclose. C. Larsen has nothing to disclose. E.J. Kezirian reports grants from Inspire Medical Systems, during the conduct of the study; and equity from Nyxoah, Pillar Palatal, Cognitive Life Science, Split Rock Scientific, Gerard Scientific, Berendo Scientific and ReVENT Medical, and grants from Autonomic Technologies, outside the submitted work; and in addition, has a patent Magnap issued, a patent Endoscopic Device and System issued, a patent Head and Neck Exercise Methods pending, and a patent Airway and Airflow Factors issued. J. Hsia has nothing to disclose. S. Chia has nothing to disclose. J. Harwick has nothing to disclose. K. Strohl reports grants (as site PI for post-approval studies) from Inspire Medical Systems, during the conduct of the study; and was consultant to Sommetrics and advisor to Galvani Bioelectronics, outside the submitted work. R. Mehra reports grants (to institution, to support efforts to collect data for the ADHERE registry) from Inspire Medical Systems, during the conduct of the study; and grants from $\mathrm{NIH}$, equipment for research from Philips Respironics and Resmed, personal fees from American Academy of Sleep Medicine, and royalties from Up to Date, outside the submitted work.

\section{References}

1 Peppard $\mathrm{PE}$, Young $\mathrm{T}$, Barnet $\mathrm{JH}$, et al. Increased prevalence of sleep-disordered breathing in adults. Am J Epidemiol 2013; 177: 1006-1014.

2 Remmers JE, de Groot WJ, Sauerland EK, et al. Pathogenesis of upper airway occlusion during sleep. J Appl Physiol Respir Environ Exerc Physiol 1978; 44: 931-938.

3 Strohl KP, Saunders NA, Feldman NT, et al. Obstructive sleep apnea in family members. N Engl J Med 1978; 299: 969-973.

4 Eckert DJ. Phenotypic approaches to obstructive sleep apnoea - new pathways for targeted therapy. Sleep Med Rev 2018; 37: 45-59.

5 Dempsey JA, Veasey SC, Morgan BJ, et al. Pathophysiology of sleep apnea. Physiol Rev 2010; 90: 47-112.

6 Verse T, Dreher A, Heiser C, et al. Leitlinie: „HNO-spezifische Therapie der obstruktiven Schlafapnoe bei Erwachsenen" Kurzfassung ArGe Schlafmedizin der Deutschen Gesellschaft für Hals-Nasen-Ohren-Heilkunde, Kopf- und Hals-Chirurgie. [S2e-guideline: "ENT-specific therapy of obstructive sleep apnea in adults" short version: Sleep Medicine Task Force of the German Society for Otorhinolaryngology, Head and Neck Surgery]. HNO 2016; 64: 310-319.

7 Strollo PJ Jr, Soose RJ, Maurer JT, et al. Upper-airway stimulation for obstructive sleep apnea. N Engl J Med 2014; 370: 139-149.

8 Woodson BT, Gillespie MB, Soose RJ, et al. Randomized controlled withdrawal study of upper airway stimulation on OSA: short- and long-term effect. Otolaryngol Head Neck Surg 2014; 151: 880-887.

9 Heiser C, Hofauer B. Predictive success factors in selective upper airway stimulation. ORL J Otorhinolaryngol Relat Spec 2017; 79: 121-128.

10 Steffen A, Sommer JU, Hofauer B, et al. Outcome after one year of upper airway stimulation for obstructive sleep apnea in a multicenter German post-market study. Laryngoscope 2018; 128: 509-515.

11 Heiser C, Thaler E, Boon M, et al. Updates of operative techniques for upper airway stimulation. Laryngoscope 2016; 126: Suppl. 7, S12-S16.

12 Heiser C, Thaler E, Soose RJ, et al. Technical tips during implantation of selective upper airway stimulation. Laryngoscope 2018; 128: 756-762.

13 Heiser C, Knopf A, Hofauer B. Surgical anatomy of the hypoglossal nerve: a new classification system for selective upper airway stimulation. Head Neck 2017; 39: 2371-2380.

14 Iber C, Ancoli-Israel S, Chessonn A, et al. The AASM Manual for the Scoring of Sleep and Associated Events: Rules, Terminology and Technical Specifications. Westchester, IL, American Academy of Sleep Medicine, 2007.

15 Sher AE, Schechtman KB, Piccirillo JF. The efficacy of surgical modifications of the upper airway in adults with obstructive sleep apnea syndrome. Sleep 1996; 19: 156-177. 
16 Budhiraja R, Parthasarathy S, Drake CL, et al. Early CPAP use identifies subsequent adherence to CPAP therapy. Sleep 2007; 30: 320-324.

17 Ancoli-Israel S, Kripke DF, Klauber MR, et al. Sleep-disordered breathing in community-dwelling elderly. Sleep 1991; 14: 486-495.

18 Irvine LE, Yang Z, Kezirian EJ, et al. Hyoepiglottic ligament collagen and elastin fiber composition and changes associated with aging. Laryngoscope 2018; 128: 1245-1248.

19 Fei T, Polacco RC, Hori SE, et al. Age-related differences in tongue-palate pressures for strength and swallowing tasks. Dysphagia 2013; 28: 575-581.

20 Van de Heyning PH, Badr MS, Baskin JZ, et al. Implanted upper airway stimulation device for obstructive sleep apnea. Laryngoscope 2012; 122: 1626-1633.

21 Vanderveken OM, Maurer JT, Hohenhorst W, et al. Evaluation of drug-induced sleep endoscopy as a patient selection tool for implanted upper airway stimulation for obstructive sleep apnea. J Clin Sleep Med 2013; 9: $433-438$.

22 Vroegop AV, Vanderveken OM, Boudewyns AN, et al. Drug-induced sleep endoscopy in sleep-disordered breathing: report on 1,249 cases. Laryngoscope 2014; 124: 797-802.

23 Heiser C, Knopf A, Bas M, et al. Selective upper airway stimulation for obstructive sleep apnea: a single center clinical experience. Eur Arch Otorhinolaryngol 2017; 274: 1727-1734.

24 Sawyer AM, Gooneratne NS, Marcus CL, et al. A systematic review of CPAP adherence across age groups: clinical and empiric insights for developing CPAP adherence interventions. Sleep Med Rev 2011; 15: 343-356.

25 Weaver TE, Grunstein RR. Adherence to continuous positive airway pressure therapy: the challenge to effective treatment. Proc Am Thorac Soc 2008; 5: 173-178.

26 Shapiro GK, Shapiro CM. Factors that influence CPAP adherence: an overview. Sleep Breath 2010; 14: 323-335.

27 Poulet C, Veale D, Arnol N, et al. Psychological variables as predictors of adherence to treatment by continuous positive airway pressure. Sleep Med 2009; 10: 993-999.

28 Chai-Coetzer CL, Luo YM, Antic NA, et al. Predictors of long-term adherence to continuous positive airway pressure therapy in patients with obstructive sleep apnea and cardiovascular disease in the SAVE study. Sleep 2013; 36: 1929-1937.

29 Woehrle H, Graml A, Weinreich G. Age- and gender-dependent adherence with continuous positive airway pressure therapy. Sleep Med 2011; 12: 1034-1036. 